\title{
Systematic Index
}

I. Tribe Serrolecaniini, Shinji 25

A. Genus Chaetococcus Maskell

1. C. bambusae (Maskell ) ....... 30

2. C. phragmitis (Marchal) ....... 35

3. C. sulcii (Green) ... 40

4. C. turanicus Borchsenius ...... 44

B. Genus Idiococcus

Takahashi \& Kanda ... 48

I. bambusae

Takahashi \& Kanda ... 49

C. Genus Kermicus

Newstead ......... 54

$K$. wroughtoni Newstead 55

D. Genus Porisaccus, gen. n. 56

1. P. jiuhuaensis (Wu), comb. n. ........ 58

2. P. sasae (Siraiwa), comb. n......... 61

E. Genus Serrolecanium

Shinji ........... 66

1. S. indocalamus Wu.. 69

2. S. kawaii, sp. n..... 73

3. S. takagii, sp. n. ... 77

4. S. tobai (Kuwana)... 80

F. Genus Tangicoccus

Kozár \& Walter ..... 84

T. elongatus (Tang) .... 85
II. Other Legless Mealybug

Genera ............. 89

A. Genus Acinicoccus Williams ......... 89

1. A. stipae Williams... 90

2. A. triodia Williams .. 91

B. Genus Antonina

Signoret .......... 94

1. A. australis Froggatt 97

2. A. bambusae Khalid \& Shafee .. 99

3. A. crawi Cockerell . 100

4. A. elongata Tang ... 103

5. A. graminis (Maskell) ....... 103

6. A. maritima Ayyar . 109

7. A. meghalayaensis Khalid \& Shafee .. 111

8. A. natalensis Brain . 111

9. A. pretiosa Ferris .. 113

10. A. purpurea Signoret 117

11. A. tesquorum Danzig 122

12. A. thaiensis

Takahashi....... 123

13. A. transcaucasica

(Borchsenius), comb. n. ....... 125

14. A. transvaalensis

Brain .......... 126

15. A. vera Borchsenius 127

16. A. zonata Green ... 128 
C. Genus Antoninoides

Ferris ............ 130

1. A. boutelouae (Parrott), comb. n. . 131

2. A. nortoni (Parrott \& Cockerell), comb. n. 133

3. A. parrotti (Cockerell) ....... 135

D. Genus Cypericoccus

Williams .......... 140

C. multipori Williams .. 141

E. Genus Nesticoccus Tang 144 $N$ sinensis Tang $\ldots \ldots \quad 144$

F. Genus Paludicoccus Ferris ........... 146 P. disticlium (Kuwana) . 146

G. Genus Parapaludicoccus Mamet ........... 150 P. isaloensis Mamet ... 151

H. Genus Peridiococcus Williams .......... 154

1. P. ethelae (Fuller) ... 155

2. P. stypheliae (Maskell) ....... 156

I. Genus Pseudantonina Green ............ 160

P. bambusae Green .... 160

J. Genus Sphaerococcus Maskell ........... 164

1. S. casuarinae Maskell 166

2. S. durus De Lotto .. 170

III. Species of Uncertain

Placement ........... 173

1. Apezococcus dakotensis (Kosztarab \& McDaniel), comb. n. 173

2. Pseudantonina aeria Williams \& Granara de Willink ....... 173
3. Pseudantonina arundinariae McConnell ...... 174

4. Pseudantonina giganticoxa Lobdell 175

5. Pseudantonina texana Ferris ...... 176

6. Sphaerococcus ferrugineus Froggatt 177

7. Sphaerococcus floccosus, nomen nudum .... 177

8. Sphaerococcus froggatti Maskell .. 178

9. Sphaerococcus melaleucae Maskell 178

10. Sphaerococcus morrisoni Fuller ... 179

11. Sphaerococcus morrisoni var. elongatus Fuller ... 180

12. Sphaerococcus newmanni Froggatt ....... 180

13. Sphaerococcus pustulans Green ... 181

14. Sphaerococcus rugosus Maskell ... 182

15. Sphaerococcus rugosus var. elongatus Maskell .. 182

16. Sphaerococcus socialis Maskell _.. 183

17. Sphaerococcus tepperi Fuller .... 183

18. Sphaerococcus tormentosus Fuller . 184

19. Sphaerococcus turbinatus Froggatt . 185 